\title{
Über die Umwandlungen des Kieselsäureanhydrids bei höheren Temperaturen.
}

\author{
Von \\ K. Endell und R. Rreise.
}

Mit 2 Figuren im Text.

In der Natur kommt das kristallisierte Kieselsäureanhydrid in drei bekannten polymorphen Modifikationen vor, dem Quarz, Cristobalit und Tridymit. Eine jede dieser Formen wandelt sich bei höheren Temperaturen wieder in eine andere um, auch gehen sie zum Teil ineinander über. Die bisher vorliegenden Daten lassen sich kurz in folgender Weise wiedergeben.

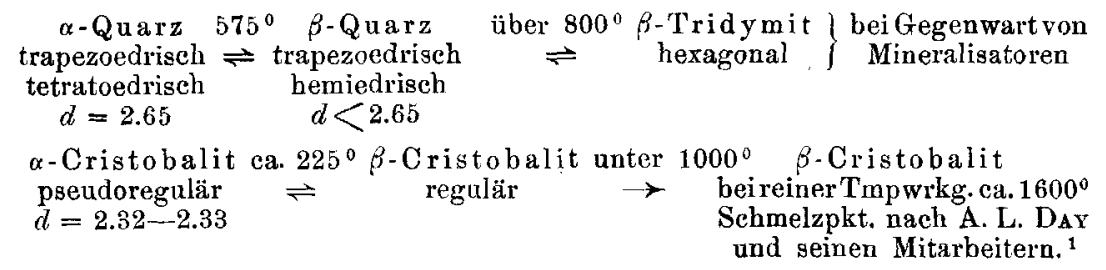

$\alpha$-Tridymit ca. $130^{\circ} \beta$-Tridy mit | ca. $1550^{\circ}$ Schmelzpkt. n. P.D.Quensed ${ }^{2}$ pseudohexagonal $\rightleftharpoons$ hexagonal $\}$ ca. $1575^{\circ}$ Schmel zpkt. nach C.DoEırer. ${ }^{3}$ $d=2.31-2.32$

In der Natur können $\alpha$-Quarz, $\alpha$-Cristabalit und $\alpha$-Tridymit nebeneinander vorkommen. Die beiden letztgenannten Formen dürften sich dann im labilen Gleichgewicht befinden. Wegen des Auftretens solcher labiler Formen und wegen der häufig vorkommenden Überschreitungen der jeweiligen Zustandsfelder ist die Aufstellung eines vollständigen Zustandsdiagramms des Systems $\mathrm{SiO}_{2}$ zurzeit nicht möglich. Die Beziehungen des Tridymits zum Cristobalit sind nicht geklärt. Die Bildung des Tridymits scheint an die Anwesenheit von Mineralisatoren gebunden zu sein. Rückbildungen des Quarzes aus Tridymit sind in der Natur beobachtet und auch im Laboratorium bewirkt worden.

1 A. L. D

2 P. D. Quensel, Centrlbl. f. Min. 1906, 657.

${ }^{3}$ C. Doelter, Physikal. Chem. Min. 1905, 100. 
Neben diesen Formen gibt es in der Natur noch faserige Varietäten der kristallisierten Kieselsäure bzw. kristalline Aggregrate, die oft unter dem Namen Chalcedon zusammengefaBt werden. Mineralogen haben nach optischen Eigenschaften verschiedene Arten wie Chalcedon, Quarzin, Lutecit und Lutecin unterschieden. Es ist jedoch auch schon öfters die Ansicht ausgesprochen worden, daB diese faserigen Varietäten nur durch ihre Struktur vom Quarz verschieden sind (F. Walígant, ${ }^{1}$ J. Beckenkamp, R. Brauns, ${ }^{3}$ H. Hein, ${ }^{4}$ O. MÜGge, ${ }^{5}$ H. Schneiderhöhn ${ }^{6}$.)

Auf die große Anzahl von Untersuchungen, die über Umwandlungen von $\mathrm{SiO}_{2}$ bei höheren Temperaturen angestellt worden sind, kann hier nicht eingegangen werden. Die Untersuchungen wurden von verschiedenen Forschern mit verschiedenen Materialien und nach verschiedenen Methoden ausgeführt. Manchmal widersprechen sich die Ansichten, so daß noch keineswegs Klarheit über dies in der festen Erdrinde am weitesten verbreitete Oxyd herrscht.

In den nachstehenden Untersuchungen haben wir uns darauf beschränkt, die reine Temperaturwirkung bei Abwesenheit von Mineralisatoren auf die verschiedenen $\mathrm{SiO}_{2}$ - Materialien $z u$ studieren. $\mathrm{Da}$ es zurzeit nicht möglich scheint, den Tridymit auf diese Weise zu erhalten, so fällt er auBerhalb des Bereichs dieser Untersuchung.

\section{Ausgangsmaterialien.}

Wenn man die vorhandenen Angaben der Literatur vergleicht, so wird man meist vergeblich nach einer näheren Bezeichnung des verwandten Quarzes suchen. Folgendes Beispiel sei angeführt. G. RosE ${ }^{7}$ hatte Tridymit erhalten, indem er feines Quarzpulver von der Dichte 2.65 der Hitze des Porzellanofens aussetzte, wobei der Quarz nach seinen Angaben in eine Modifikation von der Dichte 2.32 überging, die sich mikroskopisch als Tridymit ${ }^{8}$ erkennen ließ.

1 F. Wallérant, Bull. soc. min. de France 20 (1897), 52.

2 J. Beckenkamp, Zeitschr. f. Kristallogr. 34 (1901), 582.

3 R. Brauns, N. Jahrb. f. Min. 21 (1906), 447-468; Zentralbl. f. Min. 1911, Nr. 15 .

* H. Hers, N. Jahrb. f. Min. 25 (1908), 182-231.

○. MüqaE, Centrlbl. f. Min. 1911, Nr. 7.

' H. SohneiderhöHN, N. Jahrb. f. Min. 2 (1912), 1--32.

: G. Rose, sitz.-Ber. d. kgl. preub. Akad. d. Wissenschaft. Berlin 1869.

s Da keine Mineralisatoren zugegen waren, dürfte es Cristobalit gewesen sein. 
Seine Versuche wurden in neuerer Zeit von A. L. DAY ${ }^{1}$ bestätigt, der ebenfalls Cristobalit erhielt, wenn er feingepulverten Quarz einige 'Tage auf $1200^{\circ}$ erhitzte. R. SchWARZ ${ }^{2}$ gelang es nicht, auf diese Weise Cristobalit zu erhalten. Auch wir konnten beobachten, dab das Pulver eines bestimmten Quarzes nach viermaligem Erhitzen im Porzellanofen (= ca. 24 Stunden über 1400') im spezifschen Gewicht nur wenig abgenommen hatte, während ein anderes gleich reines Quarzpulver bereits nach ein- bis zweimaligem Erhitzen fast vollständig in Cristobalit umgewandelt war. Die nähere Untersuchung zeigte, daß das erste Pulver von einem einfachen Quarzkristall, das andere von einem stark verzwillingten Quarz stammte.

Als Quarz verwandten wir einen größeren einheitlichen Kristall von St. Gotthard, verzwillingte Quarze aus Pegmatitgängen in Saetersdalen (Norwegen) und stark verzwillingten pseudomorphen Quarz aus Gängen im Taunus. ${ }^{3}$ Die Reinheit dieser Quarze betrug nach unseren Analysen 99.8-99.9\% $\mathrm{SiO}_{2}$. Als faserige kristallisierte Form der Kieselsäure verwandten wir Chaleedon und Quarzin aus Minas Geraes (Brasilien). Der Glühverlust der benutzten Probe betrug $1 \%$, der Kieselsäuregehalt der wasserfreien Substanz 99.8 bis $99.86 \%$. Da natürlicher Cristobalit nur sehr selten in der Natur vorkommt, benutzten wir künstlichen, der durch Entglasen von Quarzglaspulver im Porzellanofen gewonnen war $\left(99,9 \% \quad \mathrm{SiO}_{2}\right)$. Ferner wurden noch sechs verschiedene Quarzgläser ${ }^{4}$ mit $0.1-1 \%$ Verunreinigungen bzw. fremden Beimengungen und amorphe Kieselsäure von Kahlbaum mit $99.3 \% \quad \mathrm{SiO}_{2}$ untersucht.

\section{Untersuchungsmethoden.}

Die genannten Ausgangsmaterialien wurden teils in $1-3 \mathrm{ccm}$ großen Stücken, die infolge Zerspringens beim wiederholten Erhitzen stets kleiner wurden, teils in Pulvern, die im Achatmörser feinst gepulvert waren, verwandt. Nach dem Erhitzen wurde das spezifische Gewicht bestimmt. Da die spezifischen Gewichte von Quarz $(2,65)$, Cristobalit (2.33) und Quarzglas (2.21) verschieden sind, so kann bereits daraus die Richtung der jeweiligen Umwandlung erkannt werden.

${ }^{1}$ A. L. Day u. Mitarbeiter, c. I. S. 184.

2 R. Sohwarz, $Z$. anorg. Chem. 76 (1912), 422.

s Dieses infolge eines falschen Gutachtens als "Geyserit" bezeichnete Material wurde kürzlich von H. SchneIderhöнs I. c. eingehend optigch untersucht.

${ }^{4}$ Vgl. darüber R. Rreke n. K. EndenL, Archiv f. d. physikal. Chemie des Olases u. d. keramischen Massen. Dez. 1912. 
Als Öfen dienten bis $800^{\prime \prime}$ Nickeldrahtwiderstandsöfen, bis $1350^{\circ}$ ein Platinfolionfen von Heraeus, beide mit rein oxydierender Atmosphäre. Für Temperaturen von $1400-1600^{\circ}$ erwiesen sich die elektris chen Kohlegrieswiderstandsöfen nach Simonis-RIEkE ${ }^{1}$ als sehr brauchbar. Wegen der in diesen Öfen herrschenden schwach reduzierenden Atmosphäre waren die Thermoelemente durch verschiedene Rohre aus glasierter MaRQUaRDTscher Masse geschützt.

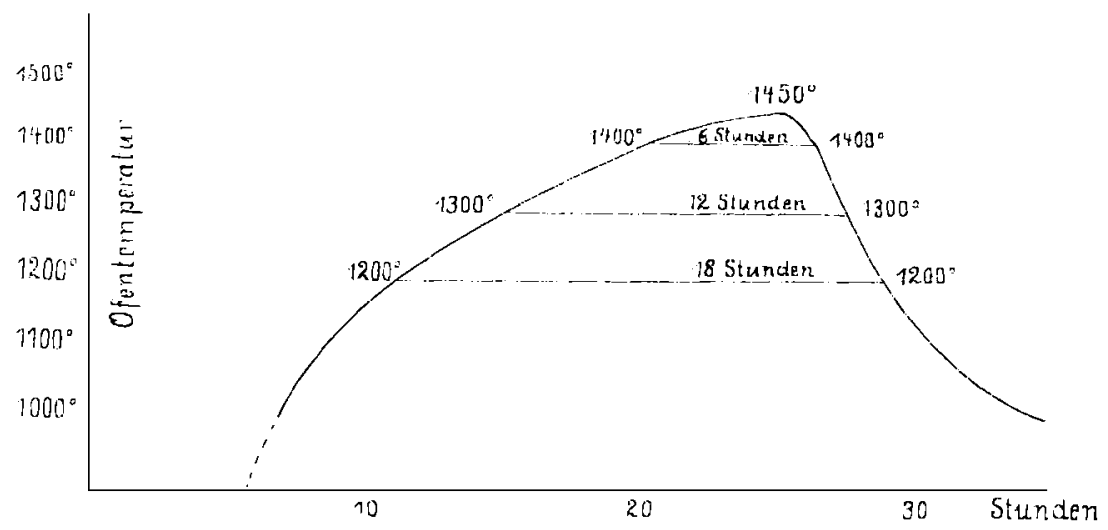

Fig. 1.

Temperatur-Zeitkurve eines Porzellanofenbrandes.

Die 'T'emperaturen wurden mittels Platin-Platinrhodium-Thermoelementen unter Anbringung der dabei üblichen Korrekturen gemessen und innerhalb der jeweils gewünschten Zeiträume konstant gehalten. Die Temperaturschwankungen betrugen bis $1300^{\circ} \pm 5^{\circ}$. von $1300-1500^{\circ} \pm 10^{\circ}$, bei $1600^{\circ} \pm 20^{\circ}$. Da die Proben in diesen Öfen böchstens zwei Stunden hintereinander erhitzt werden konnten, clie Umwandlung des Quarzes in Cristobalit aber selbst bei $1300^{\circ}$ bis $1400^{\circ}$ noch recht langsam vor sich geht, so wurden die meisten Proben auch zu wiederholten Malen der Hitzeeinwirkung des Porzellanofens ausgesetzt.

In der Literatur ${ }^{2}$ finden sich zum Teil falsche Angaben über die Temperaturen, die im Porzellanofen erreicht werden. Aus diesem Grunde und weil später die Einwirkung von ''enperatur und Zeit

${ }^{1}$ M. Simonis u. R. Rreke, Zeitschr, angew. Cherr. 1906, 1231-1233.

${ }^{2}$ Nach H. Wichelians, Vorlesungen über chemische Technologie, 2. Aufl., (1906), 386, betrïgt die Brenntemperatur des Porzellanofens etwa $1700^{\circ}$, nach Gmelis-Kraut (Handib. d. anorgan. Chemie, 67. Lieferung, Bd. III, Abt. 1, S. 136, 1908 ) sogar etwa $2000^{\circ}$, während in Wirklichkeit nur etwa $1450^{\circ}$ erreicht wird. 
auf die Umwandlungen in Porzellanofenbränden dargestellt werden wird, sei hier eine typische Temperatur-Zeit-Kurve eines Porzellanofenbrandes wiedergegeben, die mittels selbstregistrierendem PlatinPlatinrhodium-Thermoelement in den Öfen der Königlichen Porzellanmanufaktur zu Berlin aufgenommen wurde. Die Temperatur befindet sich also ca. 18 Stunden über $1200^{\circ}$, davon ca. 12 Stunden über $1300^{\circ}$ und davon ca. 5-6 Stunden über $1400^{\circ}$. Nach dem Erreichen der Maximaltemperatur von rund $1450^{\circ}$ fällt die Temperatur sehr rasch. Die Temperaturen unterhalb $1000^{\circ}$ sind in der Figur fortgelassen, da sie für die Umwandlungen der Kieselsäure kaum in Betracht kommen.

Für die Temperaturen oberhalb $1600^{\circ}$ konnten wir den Iridiumofen der Physikalich-Technischen Reichsanstalt in Charlottenburg benutzen.

Nach dem plötzlichen Herausnehmen der Pulver aus den Öfen (mit Ausnahme des Porzellanofens) wurde das spezifische Gewicht pyknometrisch bestimmt. Die Schwebemethode konnte nicht angewandt werden, da es sich um Pulver mit verschieden schweren Teilchen handelte und es gerade darauf ankam, das Bausch-Spezifische Gewicht zu kennen. Beträgt z. B. das spezifische Gewicht des rohen Quarzglases 2.21 und das des Cristobalits 2.33, so kann aus jedem dazwischen liegenden Wert berechnet werden, wieviel Cristobalit sich aus Quarzglas gebildet hat. Wenn die feinen Pulver vor dem Einfüllen in die Pyknometer gut ausgekocht werden, so werden die außen anhaftenden Luftblasen fast ganz entfernt. Vorversuche, bei denen rohes Quarzglas in Stücken nach der Schwebemethode und Quarzglaspulver pyknometrisch bestimmtwurden, zeigten Unterschiede von \pm 0.002 . Obwohl die spezifischen Gewichte möglichst sorgfältig in kleinen Pyknometern bestimmt wurden, so kamen bei Kontrollbestimmungen doch Fehler bis zu \pm 0.0025 vor, was z. B. bei der Umwandlung von Quarzglas in Cristobalit bereits einen Fehler von $\pm 2.5 \%$ bedeutet.

Erheblicher kann schon der EinfluB der KorngröBe auf die Umwandlung sein, wie wir früher am Spodumen zeigen konnten. ${ }^{1}$ Dem glaubten wir dadurch vorbeugen zu können, daß wir bei Pulvern überhaupt nur sehr feine Körner verwandten, die sich wohl bei den verhältnismäBig langen Erhitzungszeiten annähernd gleichmäßig verhalten haben. Bei der nachstehend gegebenen Figur

1 K. Endell u. R. RIEke, Z. anorg. Chem. $7 \pm$ (1912), 37-41. 
kommt es auch mehr auf die Richtung der Kurven an als auf die absolut genauen Zahlenwerte der spezifischen Gewichte.

Bei der Umwandlung des Quarzes in Cristobalit, kann aus dem Wert des spezifischen Gewichts nicht direkt auf die gebildete Menge in Prozenten geschlossen werden. Bereits bei der $\alpha \rightleftharpoons \beta$ Umwandlung bei $575^{\circ}$ wird der Quarz von feinen Sprüngen durchsetzt, die bei wiederholtem Erhitzen zunehmen. Die Umwandlung in Cristobalit, die mit einer Volumzunahme von rund $14 \%$ verbunden ist, begünstigt von neuem die Zertrümmerung. Schließlich sind die Endprodukte so stark mit mikroskopisch feinen Sprüngen durchsetzt, die auch noch đie feinsten Pulverkörner erfüllen, so daB das spezifische Gewicht zu niedrig ausfällt. Besonders auffällig ist diese Erscheinung bei der faserigen Form, dem Chalcedon, der bereits im rohen Zustand eine von feinsten Poren durchsetzte Struktur zu besitzen scheint.

Diese sehr feinen Sprünge wirken auch auf die mikroskopische Untersuchung störend ein. Infolge der an den Grenzflächen auftretenden Totalreflexion erscheinen die bei ca. $1450^{\circ}$ gebrannten Quarze und Chalcedone so gut wie optisch-isotrop und wurden auch schon wiederholt dafür gehalten. ${ }^{1}$ Bei sehr genauer Prüfung kann jedoch an günstigen Körnern am Rand die schwache Anisotropie wahrgenommen werden. Zugleich mit der körperlichen Dichte ändert sich auch die optische, so daß die Bestimmung des Brechungsindex zur Diagnostizierung der Endprodukte herangezogen werden kann. Nicht umgewandelter Quarz $(n=1.54)$ ist leicht neben Cristobalit $(n=1,485)$ zu erkennen. Die Stärke der Doppelbrechung konnte wegen der Feinheit der Umwandlungsprodukte nur geschätzt, nicht gemessen werden. Infolge der starker Verzwilligung des Cristobalits konnten auch keine kristallographischen Bestimmungen ausgeführt werden. Da der Cristobalit bei $225 \ldots 230^{\circ}$ optisch-isotrop, regulär wird, so kann im Erhitzungsmikroskop diese Umwandlung beobachtet werden, wozu sich besonders die aus Quarzglas oder Quarz bei $1600^{\circ}$ entstehenden Cristobalite eignen.

Diese sonst so bequeme optische Untersuchungsmethode versagt bei den aus Quarz bei ca. $1450^{\circ}$ gebildeten Cristobaliten, die so mit feinen Sprüngen durchsetzt sind, daß sie in der Luft fast optisch-

1 H. ie Chatedier, Compt. rend. 15. Juli 1890. - M. Herschrowitson, Zeitschr. phys. Chem. 46 (1903), 412. - I. P. HoLmquist, Geol. förening. Förhandl. April 1911. - K. ENDELL, Stahl u. Eisen 1912, 392-397. 
isotrop erscheinen. Es wurde daher auch versucht, die Wärmetönung bei der Zustandsänderung zu bestimmen, die trotz ihrer Kleinheit mittels Eisen-Konstantan-Thermoelementen wahrgenommen werden konnte.

Mehr Erfolg hatte die Verwendung einfacher Quecksilberdilatometer mit elektrischer $W$ iderstandsheizung, mit denen die Volumveränderung bei der Umwandlung ermittelt werden konnte. Da diese, wie wir fanden, beim Cristobalit bei ca. $230^{\circ}$ recht erheblich ist, so gestattet die dilatometrische Untersuchung den sichersten Nachweis des Cristobalits und ermöglicht eine einwandsfreie Unterscheidung von Tridymit, dessen spezifisches Gewicht, Brechungsindex sowie Löslichkeit in Natronlauge und Flußsäure ${ }^{1}$ fast die gleichen sind.

\section{Experimentelle Untersuchungen in dem Temperaturbereich von $900-1700^{\circ}$.}

Die Umwandlung des Quarzes in Cristobalit ist außer von der Höhe der Temperatur und der Korngröße auch von der Erhitzungsdauer abhängig. Eine Mindesttemperatur, bei der Quarz durch reine Temperaturwirkung in Cristobalit übergeht, kann wegen der Trägheit der Umwandlung kaum angegeben werden. P. D. QUENSEL, ${ }^{2}$ A. L. DAY und seine Mitarbeiter ${ }^{3}$ sowie A. BRUN ${ }^{4}$ haben unter $\mathrm{Zu}$ hilfenahme von FluBmitteln wie Lithium-Kalium-Chlorid und Natriumwolframat nachgewiesen, $\mathrm{daB}$ aus amorpher Kieselsäure bis etwa $750^{\circ}$ stets Quarz, oberhalb $850^{\circ}$ stets Tridymit entsteht. Als Umwandlungstemperatur des Quarzes in Tridymit wurde ca. $800^{\circ}$ angenommen. Als später das aus Quarz bei $1500^{\circ}$ erhaltene Umwandlungsprodukt von A. LACROIX als Cristobalit identifiziert wurde, ersetzten die Amerikaner in ihren Diagrammen stets Tridymit durch Cristobalit. Nach noch nicht abgeschlossenen eigenen Versuchen und zahlreichen Literaturangaben scheint es jedoch zweifèlhaft, ob bei Gegenwart von $\mathrm{CaO}$ und $\mathrm{Al}_{2} \mathrm{O}_{3}$ oberhalb $800^{\circ}$ wirklich stets nur Cristobalit entsteht. Die schönen Tridymitzwillinge, die in den Quarzziegeln vorkommen, sprechen eher dagegen. Diese Quarzziegel, die aus Quarziten mit $2 \%$ Kalkmilch hergestellt werden, enthalten

1 R. SCHWARz, l. c.

${ }^{2}$ P. D. Quensel, l. c.

${ }^{3}$ A. L. D ${ }_{\text {AY }}$ und Mitarbeiter, l. c.

${ }^{4}$ A. Brun, Archiv sciences phys. et nat. Genf. Juni 1908. 
ca. $95 \% \mathrm{SiO}_{2}, 2-3 \% \mathrm{Al}_{2} \mathrm{O}_{3}+\mathrm{Fe}_{2} \mathrm{O}_{3}$ und $2 \% \mathrm{CaO}$. In gleicher Weise bilden sich aus ähnlich zusammengesetzten Quarziten nach mehrmaligem Erhitzen im Porzellanofen keilförmige Tridymitzwillinge. Eine dilatometrische Prüfung eines fast vollständig aus Tridymiten bestehenden Quarzziegels zeigte keinen Effekt bei $230^{\circ}$, so daB eine Verwechslung mit Cristobalit nicht anzunehmen ist. Auch im Erhitzungsmikroskop bleiben diese T'ridymite bis weit oberhalb $300^{\circ}$ doppeitbrechend. Bei sehr boher Temperatur $\left(I^{\prime}>1500^{\circ}\right)$ scheint allerdings Cristobalit auch in Gegenwart einiger Prozent $\mathrm{Fe}_{2} \mathrm{O}_{3}, \mathrm{Al}_{2} \mathrm{O}_{3}$ und $\mathrm{CaO}$ aufzutreten, was aus den angeschmolzenen und völlig in Cristobalit umgewandelten Quarzziegeln zu schließen ist. ${ }^{1}$

Mineralisatoren wirken beschleunigend auf die Umwandlung ein. So gelingt es z. B. nicht, durch reine Temperaturwirkung amorphe Kieselsäure in Quarz umzuwandeln. Wir wählten daher $900^{\circ}$ als niedrigste Temperatur für unsere Dauerversuche. Die mit den verschiedenen Materialien in dem Temperaturbereich von $1450-1700^{\circ}$ erbaltenen Ergebnisse sind in folgender Tabelle wiedergegeben.

(Siehe Tabelle 1, S. 247.)

Unwandlungsgeschwindigkeit verschiedener $\mathrm{SiO}_{\mathrm{O}}$-Formen bei Porzellanofenhitze. (Maximal $1450^{\circ} \mathrm{C}$.)

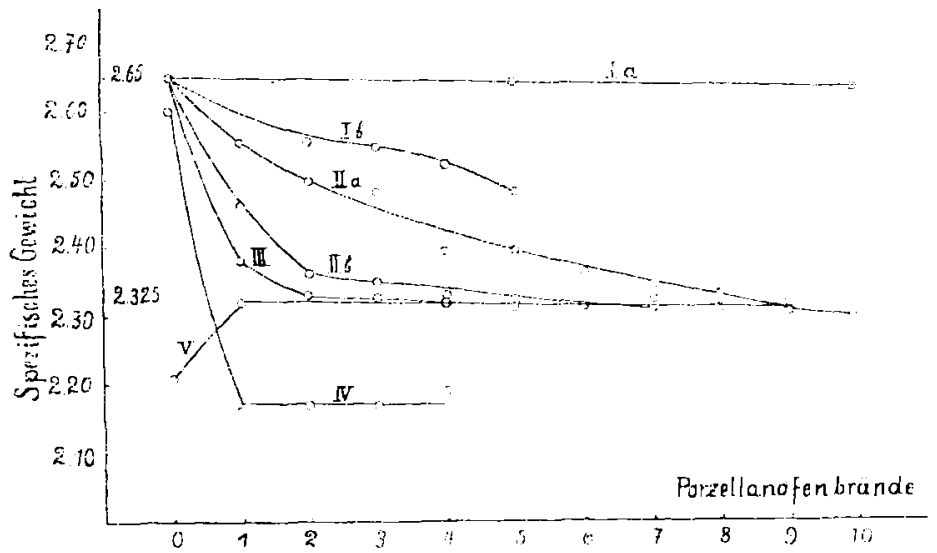

Fig. 2.

I a. Bergkristall (St. Gotthard) einfacher Kristall in Stücken. - I b. Derselbe fein gepulvert. - II a. Pseudomorpher Gangquarz (Taunus) verzwillingt; in Stïcken. - II b. Derselbe fein gepulvert. - III. Pegmatitquarz (Norwegen) verzwillingt, in Stücken. - IV. Chalcedon (Brasilieni in Stücken. - V. Kieselsäurehydrat und Quarzglaz.

I K. ENDELi, I: c. 
Cmevanllungen des Kieselsäureanhydrids bei. hïheren Temperaturen. 247

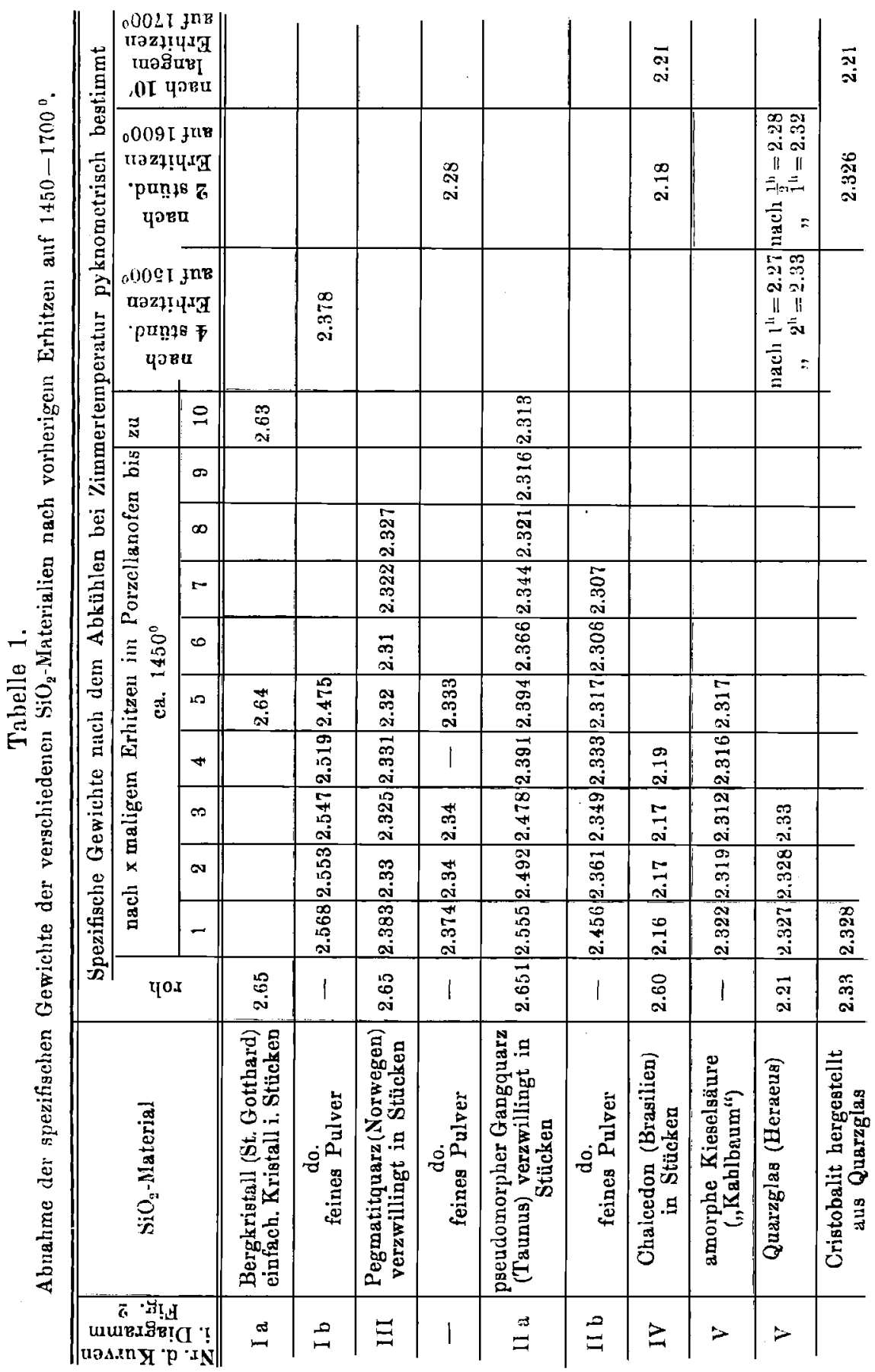


Ein Teil der Tabelle, die Wirkung wiederholter Porzellanofenbrände auf die Umwandlung, ist in Fig. 2 dargestellt.

Für die Versuche bei den niedrigeren Temperaturen wurden nur die Formen verwandt, die sich bei Porzellanofenhitze bereits nach dem ersten Brand fast vollkommen umgewandelt hatten: Quarzglas, faserige Kieselsäure (Chalcedon) und verzwillingter Pegmatitquarz in Pulverform. Im allgemeinen konnten wir die Versuche von A. L. $D_{A Y}$ und seinen Mitarbeitern ${ }^{1}$ bestätigen, da $\beta$ bei längerer Erhitzung auf Temperaturen oberhalb $1000^{\circ}$ die Umwandlung des Quarzes in Cristobalit beginnt. Eine 48 stündige Erhitzung des Chalcedon- bzw. Pegmatitquarzpulvers auf nur $900^{\circ}$ bewirkte in beiden Fällen eine geringe Abnahme des spezifischen Gewichts, die allerdings in die Fehlergrenzen der Bestimmungsmethode fiel. Daraus könnte man eventuell auch auf eine Umwandlung des Quarzes in Cristobalit bereits unterhalb $1000^{\circ}$ schlieBen. Freilich brachte nach A. L. DAY eine einen Monat dauernde Einwirkung von $900^{\circ}$ auf feinsten Kieselniederschlag keine Änderung hervor. Auch wir konnten beim Quarzglas keine Veränderung feststellen. Andererseits scheint die Abnahme des spezifischen Gewichts des Quarzes auf beginnende Umwandlung hinzudeuten. G. TAMmanN ${ }^{2}$ hat folgende wahrscheinliche Regel aufgestellt, die man vielleicht hier zur Erklärung heranziehen könnte. „Wenn die instabile Form das gröBere Volumen und die kleinere Schmelzwärme hat, so tritt Stabilitätswechsel für die beiden Formen wahrscheinlicherweise nicht ein, derselbe ist aber zu erwarten, wenn für die Volumina und die Schmelzwärmen die umgekehrte Größenbeziehung besteht." Da nun Quarzglas bzw. Kieselniederschlag a]s amorphe Körper keine Schmelzwärme und zugleich ein größeres Volumen als Cristobalit besitzen, so wandeln sie sich bei Temperaturen, die nur wenig oberhalb der Umwandlungstemperatur liegen, nicht in diesen um, Quarz dagegen erfährt beim Übergang in Cristobalit eine Volumzunahme von ca. 14\%, was nach der Tammanaschen Regel den Eintritt der Umwandlung begünstigt.

Obwohl es wegen der geringen Menge des bei $1000^{\circ}$ umgewandelten Quarzes nicht möglich ist, Cristobalit eindeutig zu identifizieren, so möchten wir doch im Hinblick auf die bei höheren

1 A. L. DaY und Mitarbeiter, 1. c. S. 181.

${ }^{2}$ G. Tammane, Nachr. d. kgl. Ges. d. Wiss. Göttingen. Math. phys. Klasse 1911, 30. Juli. 
Temperaturen erfolgende Bildung auch bereits bei $1000^{\circ}$ beginnende Umwandlung des Quarzes in Cristobalit annehmen. Es scheint sich Quarz bereits sogar unterbalb $1000^{\circ}$ bei genügend langer Hitzeeinwirkung in Cristobalit umzuwandeln, und zwar obne Mitwirkung von Mineralisatoren.

Je mehr die Umwandlungstemperatur überschritten wird, um so rascher bildet sich Cristobalit, infolge der ab. nehmenden Stabilität des Quarzes bzw. der amorphen Kieselsäure. Dies läßt sich am besten bei der Bildung des Cristobalits aus Quarzglas verfolgen. Die Menge des in der Zeiteinheit gebildeten Cristobalits nimmt von $1300^{\circ}$ an bis zu ca. $1600^{\circ}$ sehr rasch zu. Innerhalb des Temperaturbereiches von $1200-1600^{\circ}$ scheint diese Bildung des Cristobalits aus Quarzglas eine lineare Funktion der Erhitzungsdauer zu sein. ${ }^{1}$

Was sich beim Quarzglas so gut verfolgen läßt, trifft auch bei der Cristobalitbildung aus Quarz zu. Die Temperatur des Porzellanofens (maximal 1450 ) ist insofern sehr günstig für die Umwandlungen, als sie die Unterschiede zwischen den einzelnen Materialien deutlich hervortreten läBt. Bei höheren Temperaturen würden diese verwischt werden. Aus Fig. 2 läBt sich folgendes ersehen.

Während der einfache Bergkristall in Stücken (Kurve Ia) selbst nach zehnmaligem Erhitzen bis auf $1450^{\circ}$ nur sehr wenig abgenommen hat, erreichen die verzwillingten Formen meist bereits nach wenigen Bränden das spezifische Gewicht des Cristobalits. Chalcedon, dessen spezifisches Gewicht im rohen Zustand 2.60 betrug, nimmt bereits nach einmaligem Brennen bis zu 2.16 ab. Fast der gleiche Wert wurde bereits früher an gebranntem Chalcedon von H. LE CHATELIER $^{2}$ (2.16) und ELSNER ${ }^{3}$ (2.18) gefunden. Unter dem Mikroskrop ist die Sustanz jedoch nicht einheitlich. Sie besteht aus sehr kleinen klaren Teilchen von geringer Doppelbrechung und etwas größeren, meist wabigen Partien, deren zellige Struktur erst bei starker Vergrößerung sichtbar wird. Die Lichtbrechung beider Teilchen beträgt 1.48 bis 1.49. Dilatometrisch tritt ein starker Effekt zwischen $220^{\circ}$ bis $240^{\circ}$ auf, der an dem gleichen Material bereits früher von H. LE CHATELIER ${ }^{2}$ gefunden worden war. Obwohl also das spezifische Gewicht des gebrannten Chalcedons erheblich geringer ist als das des Cristo-

1 Ausführlicher hierüber vgl. K. Endell und R. Rieke, Tscherm. Min. Petr. Mittlg. 1912, Dez.

${ }^{2}$ H. Le Chatelier, l. c.

${ }^{3}$ Elsner, Journ. prakt. Chem. 99 (1866), 263. 
balits (2.33), dürfte es sich doch wohl um diesen handeln. Dafür spricht auch die gleiche Schmelztemperatur des bei $1450^{\circ}$ gebrannten Cbalcedons und des Cristobalits (siehe S. 251-252). Die sehr feine poröse Struktur macht die pyknometrische Bestimmung fehlerhaft und läßt das spezifische Gewicht zu niedrig finden.

Der wiederholt erhitzte Pegmatitquarz aus Norwegen hat zwar anscheinend ein ziemlich konstantes spezifisches Gewicht, das gut auf das des Cristobalits stimmt. Unter dem Mikroskop ist das Material jedoch nicht einheitlich. Es finden sich kleine Körnchen von noch nicht umgewandeltem Quarz, schwach doppeltbrechende Teilchen, die ihrer Lichtbrechung nach für Cristobalit gehalten werden können, und große mit feinsten Sprüngen durchsetzte Massen, die infolge ihrer Poren fast isotrop aussehen. Diese drei verschieden schweren Teile, von denen die ersten schwerer, die letzten wegen der mit Luft erfüllten Porenstruktur leichter als Cristobalit sind, ergeben zusammen Werte von 2.31-2.33. Den sicheren Nachweis, daB es sich bei der fast isotrop erscheinenden Masse wirklich um Cristobalit handelt, bringt auch in diesem Falle erst die dilatometrische Prüfung, die den bekannten starken Effekt bei ca. $230^{\circ}$ zeigt. Erhitzt man diese viermal im Porzellanofen gebrannten Quarze zwei Stunden auf $1600^{\circ}$, so werden auch die noch fehlenden Quarzteilchen umgewandelt. Die Masse ist jetzt fast einheitlich und das spezifische Gewicht dementsprechend noch weiter auf 2,28 gesunken.

Völlig analog verhalten sich auch die Umwandlungsprodukte des pseudomorphen Gangquarzes aus dem Taunus. Die gröBeren Stücke zerspringen infolge der in ihnen herrschenden Spannungen bereits nach dem ersten Brand in kleinere. Auf Spannungen führt auch H. Schneidereömen ${ }^{-1}$ die optische $Z$ weiachsigkeit der diese Quarze aufbauenden Lamellen zurück.

Amorphes Kieselsäurehydrat und Quarzglas in Pulverform werden bereits durch einmaliges Erhitzen auf $1450^{\circ}$ quantitativ in Cristobalit umgewandelt.

Aus sämtlichen Materialien entsteht somit bei längerer Temperatureinwirkung und Abwesenheit von Mineralisatoren Cristobalit. Es wurde bereits bemerkt, daB dieser am sichersten durch die ziemlich starke Volumveränderung bei seiner Zustandsänderung bei ca. $230^{\circ}$ identifiziert wurde. Diese

1 H. SัHNEIDERHöh, I. c. S. 29. 
beträgt, wie wir feststellen konnten, ${ }^{1}$ mehrere Prozent (wahrscheinlich nahezu $5 \%$ ) und läßt sich am einfachsten und klarsten in folgender Weise demonstrieren. Ein möglichst klares Stück Quarzglas wird $1-2$ Stunden auf ca. $1500^{\circ}$ erhitzt und damn plötzlich aus dem Ofen genommen. Obwohl sich bei Temperaturen von $1500^{\circ}$ innerhalb 1-2 Stunden bereits ganz erbebliche Mengen Cristobalit bilden, so ist das Glas doch vollkommen klar und durchsichtig. Bei Temperaturen unterhalb dunkelster Rotglut ca. 300-400 ${ }^{\circ}$ wird es allmählich von etwa $1 / 2 \mathrm{~cm}$ langen feinen Sprüngen durchsetzt und bei $229-227^{\circ}$ plötzlich von den Ecken aus milchig und undurchsichtig. Diese Erscheinung wird von dem Auftreten unzähliger feiner Risse begleitet. ${ }^{2}$ Diese Übereinstimmung mit der Umwandlungstemperatur des Cristobalits bei ca, $230^{\circ}$ zeigt deutlich, daß man es hier nicht mit einer erst sehr spät aufgehobenen Unterkühlung, sondern mit der Umwandlung des bei $1500^{\circ}$ gebildeten $\beta$-Cristobalits zu tun hat. Es liegt hier also der merkwürdige Fall vor, daß die bei höherer Temperatur stabile Form eines Stoffes und das daraus hergestellte Glas praktisch die gleiche körperliche und optische Dichte besitzen. Analoges wurde auch am Spodumen beobachtet. ${ }^{3}$

Das aus Pegmatitquarz, Chalcedon und Quarzglas bei ca. $1450^{\circ}$ hergestellte Cristobalitpulver bleibt nach zweistündigem Erhitzen auf $1600^{\circ}$ fast ganz locker und ist nach dem Abkühlen in seinen sämtlichen Eigenschaften unverändert. Nach den Angaben von A. L. DAX und R. B. Sosman ${ }^{4}$ liegt nun der Schmelzpunkt des Cristobalits bei ca. $1600^{\circ}$. Da in unseren Öfen keine höhere Temperatur als $1600^{\circ}$ genau gemessen werden konnte, wurde in der Physikalisch-Technischen Reichsanstalt zu Charlottenburg zusammen mit F. HoFFMANx die Schmelztemperatur des Cristobalits neu bestimmt.

Als Ofen diente der Iridium-Widerstandsofen von Heräus, der mit Stickstoffspülung benutzt wurde. Die Temperatur wurde mit

${ }^{1}$ K. Endell und R. Rieke, Tscherm. Min. Petr. Mittlg. Dez. 1912.

2 Da die schädichen Folgen der Entglasung des Quarzglases bei höheren Temperaturen erst durch die Zustandsänderung des aus ihm entstandenen Cristobalits verursacht werden, so ist es möglich, die Lebensdauer von Quarzglasgegenständen bei höheren Temperaturen ganz erheblich zn verlängern, wenn man nur dafür Sorge trägt, daB sie niemals unter $300-400^{\circ}$ abgekühlt werden.

${ }^{3}$ K. Endeli u. R. Rteke, Z. anorg. Chem. 74 (1912), 33-47. - E. Dittler und R. Balló, Z. anorg. Chem. 76 (1912), 39-69.

4 A. L. Day u. R. B. Sosman, Z. anorg. Chem. 72 (1911), 1-10. 
einem Iridium-Iridiumruthenium-Thermoelement gemessen, das nach der Drahtmethode durch die drei Schmelzpunkte des Goldes, Palladiums und Platins geeicht war. Als Temperaturen wurden dafür die Werte von A. L. DAY und R. B. Sosman ${ }^{1}\left(1063^{\circ}, 1549^{\circ}\right.$ und $1755^{\circ}$ ) angenommen, während die $Z$ wischentemperaturen nach einer quadratischen Formel für die Abhängigkeit der Thermokraft des Elements von der Temperatur interpoliert wurden.

Die Cristobalitproben befanden sich zwischen zwei Platindeckeln unmittelbar unterhalb der Lötstelle des Thermoelements. Um beurteilen zu können, welche Temperaturdifferenzen zwischen der Stelle, wo sich der Cristobalit befand, und der Lötstelle des Thermoelements herrschten, wurde auch die Thermokraft bestimmt, die das Element zeigte, wenn sich der durchschmelzende Palladiumdraht zwischen den Platindeckeln an Stelle des Cristobalits befand. Es ergab sich, daß dort die Temperatur um $5-10^{\circ}$ höher als an der Lötstelle des Thermoelements war. Die Cristobalitproben wurden je 10 Minuten auf etwa $1650^{\circ}, 1675^{\circ}$ und $1700^{\circ}$ erhitzt und dann nach plötzlichem Abkühlen untersucht.

Die auf $1650^{\circ}$ erhitzten Pulver waren noch ziemlich locker und mikroskopisch unverändert, während sie bei $1700^{\circ}$ zu einem völlig klaren Glas geschmolzen waren. Bei $1675^{\circ}$ war der Cristobalit vom Rand des Platindeckels aus zu etwa einem Drittel geschmolzen, in der Mitte aber noch unverändert. $1675^{\circ}$ liegt also sehr nahe bei der Schmelztemperatur. Unter Anbringung aller Korrekturen dürfte die Schmelztemperatur des $\beta$-Cristobalits bei $1685^{\prime \prime}$ anzusetzen sein, wobei die Fehler der Temperaturmessung \pm 10 nicht überschreiten.

\section{Folgerungen."}

Die vorstehenden Untersuchungen haben ergeben, daß die Umwandlungsgeschwindigkeit einfacher, verzwillingter und faseriger Quarze bei ca. $1450^{\circ}$ mit zunehmender Oberfläche wächst. Sie ist daher bei der fasserigen Form am gröBten. Die Tatsache, daß verzwillingte Quarze sich schneller umwandeln als einfache Kristalle,

${ }^{1}$ A. L. DaY u. R. B. Sosman, l. c.

${ }^{2}$ In dieser Arbeit werden nur die mineralogischen bzw. physikalisehchemischen Folgerungen aus vorstehenden Untersuchungen diskitiert, an anderer Stelle (Arch. f. d. physikal. Chem. d. Glases u. d. keramischen Massen 1913) wird auf die keramische Bedeutung dieser Versuche eingegangen. 
dürfte dafür sprechen, daß bei den $Z$ willingen die freie wirksame Oberfläche größer ist als bei einem gleichgroßen einfachen Kristall. Theoretisch hat P. PawLow ${ }^{1}$ nachzuweisen gesucht, daß die freie Energie von Zwillingen, die eine Funktion der freien Oberfiäche ist, größer ist als die eines einfachen Kristalls von gleicher Masse. Aus seinen Beobachtungen bei der Ätzung von Zwillingsgrenzen glaubt auch $O$. MüGGE ${ }^{2}$ schließen zu dürfen, daß sich die Grenzflächen von Zwillingsverwachsungen ähnlich wie freie Oberfläche verhalten.

Allgemein tritt nach 0 . MÜGGE ${ }^{3}$ Zwillingsbildung um so eher ein, je kürzer der Zeitraum der Kristallisation ist. Für die Quarzbildung in den Pegmatitgängen Norwegens und in den pseudomorphen Quarzgängen im Taunus darf man wohl eine verhältnismäBig rasche Kristallisation annehmen. Das gleiche dürfte für alpine Gang - und Bandquarze zutreffen. Die Untersuchungen von F. E. Wright und E. S. LarseN ${ }^{4}$ ergaben, daB die Quarze aus Graniten und Granitpegmatiten in der Regel in komplizierter Weise verzwillingt sind. 30 Plättchen aus Schriftgranit von Clar Creek, (Kalifornien), nach der Basis erwiesen sich fast sämtlich verzwillingt nach $\{10 \overline{1} 0\}$ (O. MÜGGE). ${ }^{5}$ Der eine von uns (K. E.) hatte Gelegenheit, eine größere Anzahl ron Gang- und Bandquarzen aus dem Zillertal (Tirol) zu sammeln. Die Quarze waren sehr wenig ausgebildet, häufig verzwillingt und zeigten bereits nach einmaligem Erhitzen im Porzellanofen bis zu ca. $1450^{\circ}$ eine recht erhebliche Abnahme des spezifischen Gewichts. Möglicherweise beschleunigen auch die in diesen Quarzen zahlreich vorhandenen Gas- und Flüssigkeitseinschlüsse die Umwandlung.

Nach S. CALDERON ${ }^{6}$ sind die Quarze zahlreicher Gänge sehr unvollkommen kristallisiert und stellen gewissermaßen den Übergang zwischen dem einheitlichen Quarz und einem Zustand „undeutlicher Individualisierung“ dar. Vermutlich haben sich diese Gänge rasch aus übersättigter Lösung gebildet. Erst nach Aufhebung der Über-

1 P. Pawlow, Z. phys. Chem. 72 (1910), 385.

${ }^{2}$ O. MứGE, Verhandl. Deutseh. Naturf. u. Ärzte, Münster 1912, Abt. f. Mineralogie, Diskussion nach Vortrag von K. ENDELl und persönliche Mitteilung.

"O. Müqge, Fortschritte d. Mineral. Jena 1910, S. 65 .

${ }^{4}$ F. E. Wright u. E. S. Larsen, Z. anorg. Chem. 68 (1910), 367.

ऽ O. MüGGe, Centrlbl. f. Min. 1907, 195.

'S. Caloeron, Boll. R. Soc. esp. Hist. nat. Nov. 1908, ref. Z. f. Kristallogr. 1911, $300 / 1$.

z. anorg. Chem, Ha. Th. 
sättigung kann die langsame Kristallisation das beständigste System, den einfachen Kristall, entstehen lassen. ${ }^{1}$ So kann man in alpinen Klüften oder in den bekannten Melaphyrdrusen von Idar, Theis oder Brasilien beobachten, daß die einfachen Kristalle bisweilen auf einer verschieden starken, nur wenig individualisierten Quarzschicht aufsitzen. Die verhältnismäßige Seltenheit schöner einfacher Quarzkristalle in Drusen oder Klüften gegenüber den derben, wenig individualisierten oft verzwillingten Quarzen der Quarz-Bänder und -Gänge in den Alpen scheint dafür zu sprechen, daß dort die Kristallisation aus übersättigter Lösung überwiegt.

$\mathrm{Zu}$ dem gleichen Schluß gelangte auch J. KöNIGSBERger ${ }^{2}$ auf Grund seiner Beobachtungen an Quarzbändern im Aarmassiv und durch Kristallalisationsversuche mit beißen Lösungen von Kupfersulfat und Kaliumbichromat in länglichen Glasgefäßen, die ungefähr die Form von Mineralkiüften hatten und in horizontaler Lage mit Wasser erkalteten. Die derbkristallinen Quarzmassen in Bändern dürften also aus übersättigter Eösung bei rascher Abkühlung entstehen, während die Bildung schöner Kristalle langsame Abküblung und eine verdünnte Lösung mit groBer Diffusionsgeschwindigkeit erfordert.

Die faserigen Formen der kristallisierten Kieselsäure, die sich infolge ihrer großen Oberfläche am schnellsten bei höheren Temperaturen in Cristobalit umwandeln, scheinen mit Quarz identisch zu sein, was auch ihre Zustandsänderung beweist, die von $H$. LE Chatelier ${ }^{3}$ dilatometrisch bestimmt wurde. Die Aufstellung verschiedener Formen, wie Chalcedon, Quarzin, Lucetit und Lutecin nur auf Grund optischer Eigenschaften scheint nicht gerechtfertigt. Der Begriff der ,Form" (Phase) deckt sich, wie G. TammanN" ausführt, nicht mit dem Begriff der kristallographischen Kristallform. Als "Formen" werden thermisch verschiedene Kristallformen bezeichnet, die sich betreffs ihres Volumens ihres Wärmeinhaltes und ihrer Gleichgewichtskurven voneinander unterscheiden. Solange nicht wenigstens einige dieser Daten einwandsfrei von den verschiedenen faserigen Kieselsäuren vorliegen, wird man wohl der

'Tgl. auch O. NiagL, Kolloid-Zeitsch. 1912, 268-273.

J. Königsberger, N. Jahrb. f. Min. 14 (1901), 104-106.

${ }^{3}$ H. Le Chathifier, Compt. rend. 1559, 108, 1046.

* G. Tammann, 1. c. S. $1-2$. 
Ansicht derjenigen Mineralogen beipflichten müssen, welche jene nur für Strukturvarietäten des Quarzes halten.

S. CALDERON ${ }^{1}$ vergleicht die Entwickelung der Kieselsäure vom amorphen bis zum holokristallinen Zustand mit der Entglasung einer amorphen Substanz und behauptet, die Silikate und der Schwefel böten die besten Beispiele von Kristalliten, die eine Mittelstellung zwischen Kolloid und Kristalloid einnehmen. Am Quarzglas haben G. SpezIA ${ }^{2}$ und A. BnuN ${ }^{3}$ die experimentellen Belege für diese Behauptung erbracht. Auch dem einen von uns (K. E.) gelang es, in einer mit einer Goldplatinlegierung ausgekleideten Bombe in Gegenwart von Wasser und etwas Ammoniumfluorid bei $300^{\circ}$ durch achtstündige Einwirkung Quarzglas in eine faserige Masse umzuwandeln, die in ihren optischen Eigenschaften mit Quarz übereinstimmte. A. BRUN erhielt bei vierzigstündiger Einwirkung von Alkalichloriddämpfen auf Quarzglas die gleichen Fasern. Bei längerer Dauer des Versuches vereinigten sich die Fasern zu größeren und bildeten bereits nach 3-4 Tagen ,einen optisch homogenen Kristall von $3 \mathrm{~mm}$ Länge“. Es kann kaum besser gezeigt werden, dab die faserige Form ein Durchgangsstadium zum Quarzkristall sein kann, der Übergang einer infolge ihrer großen Oberfläche instabilen Form in die stabile. J. KönigsBerger und W. MüLLER ${ }^{\star}$ haben durch Versuche in ihrer Druckbombe gezeigt, daß bei höherer $\mathrm{SiO}_{2}$-Konzentration und damit verbundener rascher Ausfüllung durch Bodenkörperreaktion hauptsächlich Chalcedon als instabiles Produkt auftrat, während sich im Filterrohr aus verdünnter Lösung wohlentwickelte Quarzkristalle bildeten. Daß übrigens Chalcedon nicht direkt aus Lösung, sondern durch Umwandlung des Hydrogels entsteht, nimmt auch $\mathrm{O}$. MÜGGE ${ }^{5}$ an.

Ein einfacher Kristall von Quarz kann sehr lange auf Temperaturen von $1300-1400^{\circ}$ erhitzt werden, ohne daB er sich in Cristobalit umwandelt. Derartige Überschreitungen der Stabilitätsgrenzen erschweren die Aufstellung eines Zustandsdiagramms. Es

3. Calderon, I. c. vgl, auch H. Vogelsana, Die Kristalliten, Bonn 1875, S. $43 \mathrm{ff}$.

2 G. Spezia, Atti Accad. Scienz. Torino 1903, 38.

s. Brun, l. c.

+ J. Könrasberaer u. W. J. Müller, Centrlbl. f. Min. 1906, S. 348.

`O. Mügae, Centrlbl. f. Mir. 1911, S. ?. 
scheint sogar möglich zu sein, den Bergkristall zu schmelzen, ohne $d a B$ er vorher in Cristobalit übergeht. Wenigstens gelang es uns wie auch bereits früher A. L. DAY und seinen Mitarbeitern bei schneller Erhitzung, ein Stück Quarz als solches zu schmeizen. Die Probe enthielt nach dem Abkühlen nur unveränderten Quarz und Quarzglas. ${ }^{1}$ Unter dem Mikroskop konnte kein Cristobalit gefunden werden. Auch O. MÜGGE ${ }^{2}$ und A. BLACKIE ${ }^{3}$ beobachteten Splitter von unverändertem Quarz und Quarzglas. Eine genaue Bestimmung des Quarzschmelzpunktes dürfte mit großen Schwierigkeiten verbunden sein. Nimmt man feines Quarzpulver, so wird es sich bei Temperaturen von $1500-1600^{\circ}$ sehr schnell in Cristobalit umwandeln und man erhält den Cristobalitschmelzpunkt. Bei der Verwendung größerer Kristallbruchstücke wird man wohl stets eine zu hohe Temperatur finden, weil die Quarzprobe infolge ihrer geringen Wärmefähigkeit eine tiefere Temperatur besitzen wird ais das Thermoelement.

Nach G. TammanN ${ }^{4}$ liegt der Schmelzpunkt der instabilen Form bei kleinen Drucken immer unterhalb des Schmelzpunktes der stabilen und gibt die Reihenfolge der Schmelzpunkte die Reibenfolge der relativen Stabilitäten an. Da Quarz oberhalb $1000^{\circ}$ instabil ist in bezug auf den stabilen Cristobalit, so müßte danach seine Schmelztemperatur unterhalb der des Cristobalits $\left(=1685^{\circ}\right)$ liegen. Vergleicht man damit die Angaben in der Literatur für den Quarzschmelzpunkt, so findet man $1600^{\circ}$ (C. Dollter), ${ }^{5}<1650^{\circ}$ (A. LaMrPeN, ${ }^{6}$ ) $1685^{\circ}$ (W. Hempel, ${ }^{7}$ ) $<1700^{\circ}$ (H. Heraeus, ${ }^{8}$ ) $1775^{\circ}$ (RoBerts Austen: $\left.{ }^{9}\right) 1780^{\circ}$ (A. BRUN ${ }^{10}$ ) usw. Da meist nähere Angaben über

1 Da der Quarz bei der technischen Herstellung des Quarzglases von sehwacher Rotglut möglichst schnell auf ca. $1800^{\circ}$ erhitzt wird, so dürfte er meist als solcher schmelzen. Die Bezeichnung "Quarzglas" ist also in gewissem Sinne gerechtfertigt.

${ }^{2}$ O. MüGge, N. Jahrb. f. Min. 1907, S. 196 u. Centrlbl. f. Min. 190s, S. 37.

3 A. B

${ }^{4}$ G. Tammann, 1. c. 5-6 und Zeitsehr. phys. Chem. 29 (1899), 67.

${ }^{5}$ C. Doeiter, l. c.

- A. Lampen, Journ. chem. Soc. 28 (1906), 846--853.

7 W. Hемpes, Z. f. Elektrochem. 9 (1903), 850.

s H. Heraeds, Intern. KongreB f. angew. Chemie, Berlin 1903. ref. Z. $f$. Elektrochem. 9 (1903), 43.

9 Robents Adstex, Phil. Mag. 1899.

10 A. Brux, Arch. science phys. nat., Genf 1902. 
den verwandten Quarz, KorngröBe und Erhitzungsgeschwindigkeit fehlen, andererseits die Temperatur nach verschiedenen Methoden gemessen wurde, so erlauben die vorliegenden Daten keinen sicheren SchluB auf den Quarzschmelzpunkt.

Nach der neuen Theorie von A. Smits ${ }^{1}$ bedeutet Allotropie Entmischung im festen Zustand. Bei den allotropen Stoffen besteht jede feste Phase im stabilen Zustand aus einem innerlichen Gleichgewicht zwischen verschiedenen Molekülarten. Ändert man die Temperatur so rapid, daB das innerliche Gleichgewicht der Temperatur nicht folgen kann, so tritt der zusammengesetzte Charakter des Stoffes zutage, wobei dann der Endschmelzpunkt höher oder tiefer liegt, als der unäre stabile Schmelzpunkt liegen wird.

Auch diese Theorie, die am Schwefel, Phosphor und Quecksilberjodid experimentell geprüft wurde, verlangt für die polymorphen Formen Quarz und Cristobalit zwei voneinander verschiedene Schmelzpunkte. Wenn auch direkte Schmelzung des Quarzes kein ausreichender Beweis ist - es könnte sich möglicherweise intermediär Cristobalit gebildet haben -, so darf man doch wohl auf Grund der an anderen Stoffen gemachten Erfahrungen annebmen, daß Quarz einen eigenen Schmelzpunkt besitzt und dieser wegen der beiderseitigen Stabilitätsbeziehungen unterhalb dessen des Cristobalits $\left(=1685^{\circ}\right)$ liegt.

G. Tammann ${ }^{2}$ unterscheidet der Stabilität nach folgende vier Klassen von polymorphen Kristallen.

1. Die total und absolut stabilen Formen. Diese sind dadurch gekennzeichnet, daß sie im ganzen Zustandsfeld, in dem sie überhaupt existenzfähig sind, absolut stabil sind.

2. Die partiell und absolut stabilen Formen. Jede Form hat ihr eigenes Zustandsfeld absoluter Stabilität, das aber nicht das ganze Gebiet ihrer Existenzfähigkeit umfaßt.

3. Die total instabilen Formen.

4. Die partiell instabilen Formen.

Versuchen wir mittels dieser Klassifikation Klarheit in die polymorphen Formen des kristallisierten Kieselsäureanhydrids zu bringen, so können wir sagen, daß $\alpha$-Quarz und vielleicht auch $\alpha$-Cristobalit zu den total und absolut stabilen Formen gehören.

${ }^{1}$ A. Sмiтs, Zeitschr. phys. Chem. 74 (1911), 421-444 u. 77 (1911), 367-87.

${ }^{2}$ G. Tammane, I. c. S. 8. 
$\beta$-Quarz besitzt von $575^{\circ}$ bis ca. $800^{\circ}$ ein Zustandsfeld absoluter Stabilität, ist aber bis $1400^{\circ}$ und höher noch existenzfähig. Er dürfte also zur zweiten Klasse gebören. Wenn man berücksichtigt, daB Cristobalit unter $800^{\circ}$ bei Zuhilfenahme von Mineralisatoren in Quarz umgewandelt werden kann, so scheint auch $\beta$-Cristobalit die Voraussetzungen der zweiten Klasse zu erfüllen. Von ca. 800 bis $1685^{\circ}$ ist er absolut stabil, von ca. $800-230^{\circ}$ jedoch partiell stabil. Unterhalb $800^{\circ}$ tritt dje Rückbildung des Quarzes aus Cristobalit ohne weiteres nicht ein, einmal wegen der geringen Reaktionsgeschwindjgkeit, die durch die große Viskosität des Cristobalits unterhalb $800^{\circ}$ bedingt ist, und dann auch wohl aus dem bereits (S. 248) angeführten Grund, weil er als die instabilere Form gegenüber Quarz das größere Volumen besitzt.

Da die Stabilitäts- und Existenzverhältnisse des Tridymits trotz der eingehenden Untersuchungen von P. D. Quenser und anderer namentlich in ihrer Beziehung zum Cristobalit noch nicht geklärt sind, so läßt sich über den Tridymit nichts Sicheres aussagen. Wahrscheinlich werden die Verhältnisse des Polymorphismus des kristallisierten Kieselsäureanhydrids bei Einbeziehung des Tridymits noch erheblich komplizierter, so daß die hier vorgenommene Übertragung des von G. Tammann aufgestellten Klassifikationsprinzips nur als ein vorläufiger Versuch aufgefaßt sein will.

\section{Ergebnisse.}

1. Einfache, verzwillingte und faserige Quarze sowie amorphe Kieselsäure in Form von Kieselsäurehydrat und Quarzglas werden bei höheren Temperaturen und Abwesenheit von Mineralisatoren in Cristobalit umgewandelt.

2. Die Umwandlungsgeschwindigkeit nimmt mit wachsender Überschreitung der unterhalb $1000^{\circ}$ gelegenen Umwandlungstemperatur zu.

3. Die Umwandlungsgeschwindigkeit der einfachen, verzwillingten und faserigen Quarze in Cristobalit bei ca. $1450^{\circ}$ ist von der Größe der Oberfläche abhängig und daher bei der faserigen Form am größten.

4. Bei genügend schneller Ërhitzung scheint sich der Schmelzpunkt des $\beta$-Quarzes realisieren zu lassen; da Quarz bei Temperaturen oberhalb $1000^{\circ}$ gegenüber Cristobalit instabil ist, so dürfte sein Schmelzpunkt unterhalb des von $\beta$-Cristobalit - also $T<1685^{\circ}$ liegen. 
Lmwandlungen des Kieselsäureanhydrids bei höheren Temperaturen. 259

5. Die endgültige Aufstellung eines Zustandsdiagramms des Systemes $\mathrm{SiO}_{2}$ ist wegen des Auftretens labiler Formen und der möglichen Überschreitung der jeweiligen Zustandsfelder zurzeit nicht möglich. Es wurde versucht, die von G. TAMmañ aufgestellte Klassifikation polymorpher Kristalle und die neue Theorie von A. Surts über die Erscheinung Allotropie auf das System $\mathrm{SiO}_{2}$ anzuwenden.

Berlin-Charlottenburg, Chemiseh-technische Versuchsanstalt bei der ligl. Pornellanmanufaltur.

Bei der Redaktion eingegangen am 9. November 1912. 